\title{
Estrategias metacognitivas en estudiantes de las Carreras de Psicología y Administración de Empresas de la Universidad Privada María Serrana, Asunción. $2018{ }^{21}$
}

\section{Metacognitive strategies in students of the Careers of Psychology and Business Administration of the María Serrana Private University, Asunción, 2018}

Gerardo Picón ${ }^{22}$; Anastacia Burgos ${ }^{23}$

\section{RESUMEN}

La metacognición posee un relacionamiento directo con los procesos de monitoreo activo y la regulación de los procesos cognitivos como un sistema de control. La metacognición resulta muy relevante para trabajar en estilos cognitivos y estrategias de aprendizaje en la medida en que el aprendiz posea conciencia de su pensamiento o procesos de aprendizaje. La investigación tuvo como objetivo evaluar las estrategias metacognitivas en los estudiantes de las carreras de Psicología y Administración de Empresas de la Universidad Privada María Serrana de Asunción, Paraguay, año 2018, identificando las estrategias cognitivas, de planificación, monitoreo y consciencia metacognitiva. Fue un estudio cuantitativo, no experimental, descriptivo transeccional utilizando como instrumento el inventario sobre estrategias metacognitivas de O’Neil y Abedi, incluyendo la población de estudiantes de las carreras de Psicología y Administración de Empresas de la Universidad Privada María Serrana, en Asunción, Paraguay, en el año 2018. Los resultados señalan que la mayoría de los estudiantes de la Carrera de Psicología siempre intentan descubrir las ideas principales o la información relevante de la tarea o actividad que realizan. Por otra parte los estudiantes de la Carrera de Administración expresan que siempre son conscientes de lo que piensan sobre la actividad de aprendizaje o problema y que comprueban su trabajo mientras lo están realizando, demarcando una conciencia metacognitiva y la aplicación de estrategias de monitoreo. Tanto los estudiantes de Psicología como de Administración indican a través del inventario que están conscientes de qué técnica o estrategia de pensamiento usan y cuándo usarla y de los procesos de pensamiento que utilizan.

Palabras clave: Metacognición; Estrategias metacognitivas; Autorregulación

\footnotetext{
${ }^{21}$ Fecha de recepción: noviembre 2018; fecha de aceptación: noviembre 2018

${ }^{22}$ Profesor titular jubilado Universidad Francisco de Miranda, Coro, Venezuela. Profesor Universidad Privada María Serrana, Asunción, Paraguay.

${ }^{23}$ Profesora jubilada del Ministerio de Educación, Coro, Venezuela.
} 


\section{ABSTRACT}

Metacognition has a direct relationship with active monitoring processes and the regulation of cognitive processes as a control system. Metacognition is very relevant to work on cognitive styles and learning strategies to the extent that the learner is aware of their thinking or learning processes. The objective of the research was to evaluate the metacognitive strategies of Psychology and Business Administration students of the María Serrana Private University of Asunción, Paraguay, 2018, identifying cognitive, planning, monitoring and metacognitive awareness strategies. It was a quantitative, non-experimental, descriptive, transectional study using as an instrument the inventory on metacognitive strategies of O'Neil and Abedi, including the population of students of the Psychology and Business Administration courses of the María Serrana Private University, in Asunción, Paraguay, in the year 2018. The results indicate that most students of the Psychology Degree always try to discover the main ideas or the relevant information of the task or activity they perform. On the other hand the students of the Administration Career express that they are always aware of what they think about the learning activity or problem and that they check their work while they are doing it, demarcating a metacognitive awareness and the application of monitoring strategies. Both students of Psychology and Administration indicate through the inventory that they are aware of what technique or strategy of thought they use and when to use it and of the thought processes they use.

Keywords: Metacognition; Metacognitive strategies; Self-regulation 


\section{INTRODUCCIÓN}

La metacognición ha sido considerada una estrategia para el desarrollo del pensamiento y del aprendizaje al profundizar en el proceso de reflexión o autoexamen de los procedimientos, técnicas y recursos de qué, cómo y cuándo aprendemos. Es parte de un proceso de concientización del propio conocimiento. Las estrategias metacognitivas permiten el repensar y enfocar un problema o una actividad de aprendizaje discriminando las técnicas, procedimientos y recursos más apropiados.

Según Flavell (1979) el conocimiento metacognitivo se fundamenta principalmente en conocimiento o creencias sobre qué factores o variables actúan e interactúan y de qué manera afectan el proceso cognitivo, destacando tres categorías principales de estos factores o variables: la persona, la tarea y la estrategia. Las variables personales refieren a lo que uno reconoce sobre sus propias fortalezas y debilidades en el aprendizaje en el procesamiento de la información; las variables de la tarea es lo que uno sabe o puede resolver comprendiendo la naturaleza de una tarea y los requerimientos de procesamiento de la información para completarla; y las variables de la estrategia son aquellos procedimientos que se aplican de manera flexible para cumplir con éxito la tarea.

"En la perspectiva cognitiva las cosas cambian radicalmente porque la enseñanza parte de problemas o temas sentidos por los alumnos, que hacen parte de su vida real y de su interés vital. Y no sólo esto. El proceso de pensar y de aprender lo alimenta el mismo aprendiz con sus conocimientos, ideas y experiencias previas acerca del problema propuesto." (Flórez, 2000)

"La metacognición se compondría, por lo tanto, de un componente de naturaleza declarativa, "saber qué" acerca de la propia actividad cognitiva, y de otro compo- nente de naturaleza procedimental, "saber cómo" aplicar los procesos cognitivos de manera eficaz en el contexto de una determinada tarea" (Pérez de Albéniz, Escolano, Pascual, Lucas y Sastre, 2015, p. 98)

Osses y Jaramillo (2008) consideran que "La importancia de la metacognición para la educación radica en que todo niño es un aprendiz que se halla constantemente ante nuevas tareas de aprendizaje. En estas condiciones, lograr que los alumnos "aprendan a aprender", que lleguen a ser capaces de aprender de forma autónoma y autorregulada se convierte en una necesidad."

Según Livingston (2003) el estudio de la metacognición ha proporcionado a los psicólogos educativos una perspectiva sobre los procesos cognitivos involucrados en el aprendizaje y lo que diferencia a los estudiantes exitosos de sus compañeros menos exitosos. También tiene varias implicaciones para realizar intervenciones durante el proceso de instrucción, tales como enseñar a los estudiantes cómo ser más conscientes de sus procesos de aprendizaje y productos, así como cómo regular esos procesos para un aprendizaje más efectivo.

Los estudios de O'Neil y Abedi (1996) señalan que la metacognición tendría cuatro dimensiones: conciencia, estrategias cognitivas, planificación y control.

En investigaciones relacionadas con la metacognición se logra encontrar en Vallejos, Jaimes, Aguilar y Merino (2012) que evaluaron la validez y confiabilidad del inventario de estrategias metacognitivas en una muestra de estudiantes universitarios de Lima y Pucallpa. El estudio se efectuó con una muestra (grupo normativo) de 687 estudiantes universitarios de las ciudades de Pucallpa (277) y Lima (410). El instrumento que se aplicó fue el "Inventario de Estrategias Metacognitivas", elaborado por O’Neil y Abedi (1996), traducido por: Martínez y con adaptación lingüística por parte de los autores. Está compuesto por 
20 ítems con escala tipo Likert, con cinco opciones de respuesta (1: Nunca; 2: Pocas veces; 3: Regular; 4: Muchas veces; 5: Siempre). En la investigación se llegó a las siguientes conclusiones: todos los ítems demuestran amplia consistencia en la evaluación de la actividad metacognitiva. Estos resultados podrían estar condicionados por la variedad y condiciones de las diferentes muestras estudiadas, lo que exigiría contar con baremos de acuerdo a diferentes condiciones sociodemográficas.

Arteta y Huaire (2016) desarrollaron también un estudio para establecer la relación que existía entre las estrategias metacognitivas y las concepciones de aprendizaje en estudiantes universitarios bajo el diseño descriptivo correlacional con una muestra constituida por 369 estudiantes del curso de Total Wellness de la Universidad San Ignacio de Loyola. Los instrumentos utilizados fueron el cuestionario de auto reporte de O'Neil y Abedi (1996) y el cuestionario de auto-reporte (CONAPRE), diseñado y validado por Martínez, 2004, que consta de 15 ítems. Se evidenció que los estudiantes de los primeros ciclos presentaban un nivel bajo de estrategias metacognitivas. Los resultados se relacionan con los reportes de Martínez (2007) y se deberían principalmente a que los encuestados pertenecían a los primeros ciclos de la Universidad, dado que en su investigación pudo evidenciar que existían diferencias entre los distintos niveles de estudio. Así, los estudiantes de final de carrera eran quienes presentaban puntuaciones significativamente superiores a diferencia de los estudiantes de los primeros ciclos. Considera que las estrategias metacognitivas son un factor que aumenta en relación con los niveles de conocimiento y los grados de formación.

Sandoval (2015) originó otra investigación cuyo objetivo fue determinar la(s) influencia(s) de los modelos de nivel de pericia y de metacognición, sobre la concepción de aprendizaje en estudiantes universitarios de psicología con la participación de 184 sujetos de ambos sexos, pertenecientes a la ciudad de Arica, Chile. Los resultados apuntaron a que el nivel de pericia y las estrategias metacognitivas correlacionaban significativamente con la concepción de aprendizaje.

Esta investigación tuvo como objetivo general evaluar las estrategias metacognitivas en los estudiantes de las carreras de Psicología y Administración de Empresas de la Universidad Privada María Serrana de Asunción, Paraguay, año 2018, identificando las estrategias cognitivas, de planificación, monitoreo y consciencia metacognitiva.

\section{MÉTODO}

Fue un estudio cuantitativo, no experimental, descriptivo transeccional utilizando como instrumento el inventario sobre estrategias metacognitivas de O'Neil y Abedi que contiene 20 ítems sobre las estrategias cognitivas, de planificación, monitoreo y consciencia metacognitiva. Se utilizó el muestreo no probabilístico abarcando toda la población de estudiantes de las carreras de Psicología y Administra- ción de Empresas de la Universidad Privada María Serrana, en Asunción, Paraguay, en el año 2018 quienes participaron voluntariamente en el estudio. La población total de estudiantes de Administración era de 92 y de Psicología 71. Los estudiantes corresponden a los inscriptos desde el primero hasta el cuarto año de las mencionadas carreras. Participaron 63 de Administración y 9 de Psicología. 


\section{RESULTADOS}

Los resultados se desarrollan conside- los estudiantes de la Carrera de Psicolorando las veinte actividades del inventario sobre estrategias metacognitivas de O'Neil y Abedi. El Gráfico 1 presenta los resultados sobre el grado de frecuencia de aplicación de las estrategias metacognitivas por gía. Se observa que mayoría selecciona la tercera actividad que intenta descubrir las ideas principales o la información relevante de dicha tarea o actividad en el grado 5 (siempre).

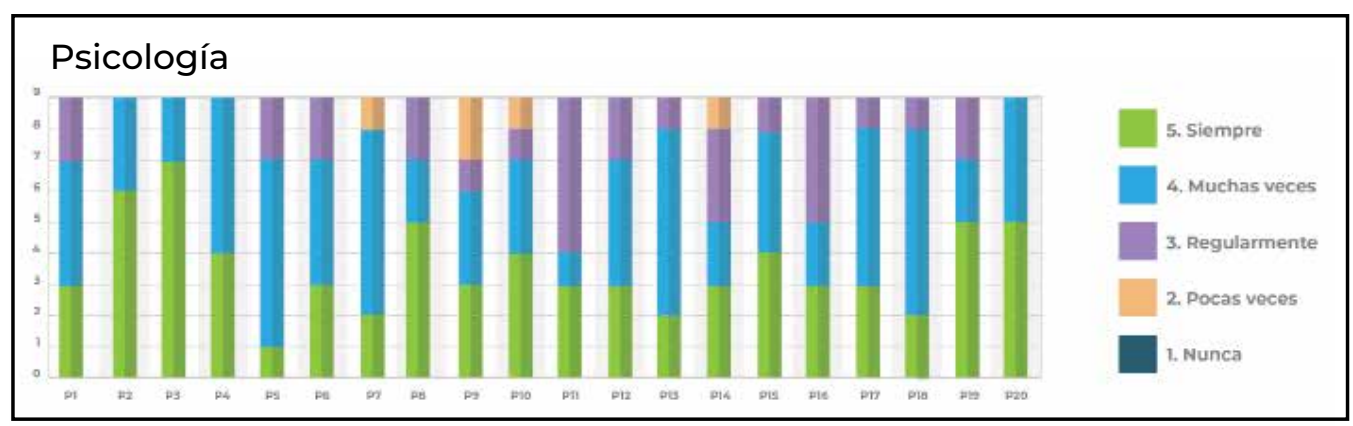

Gráfico 1. Distribución de respuestas por actividad y grado de frecuencia del inventario de estrategias metacognitivas. Carrera de Psicología.

El Gráfico 2 agrupa las actividades relacionadas con la conciencia metacognitiva que incluye las actividades $1,5,9,13 \mathrm{y}$ 17 , destacando la 5 y la 13 , lo que indica que los estudiantes muchas veces están conscientes de qué técnica o estrategia de pensamiento usan y cuándo usarla y de los procesos de pensamiento que utilizan (de cómo y en qué estás pensando).

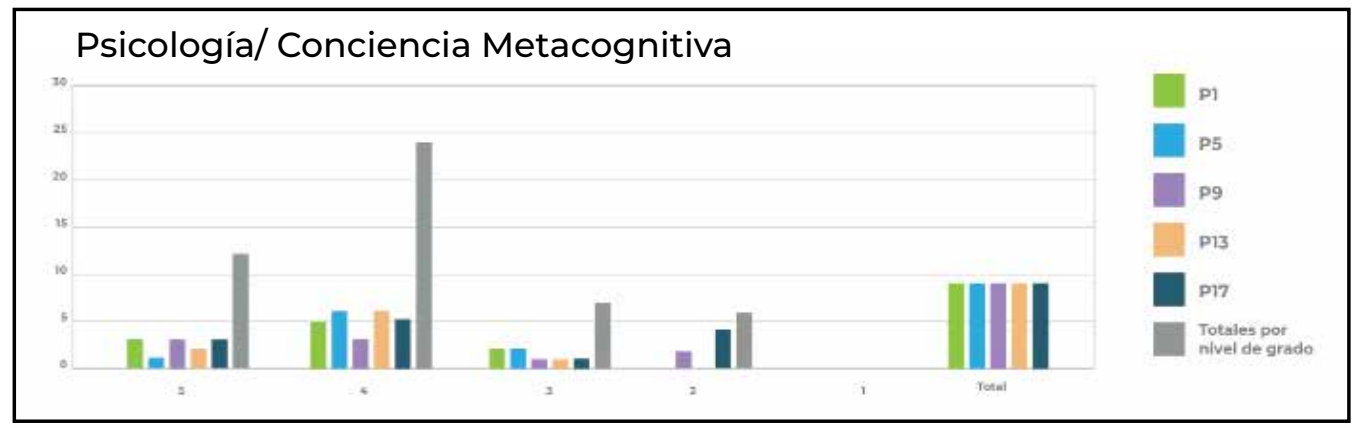

Gráfico 2. Distribución del grado de frecuencia de las estrategias de conciencia metacognitiva. Carrera de Psicología.

El Gráfico 3 agrupa las actividades relacionadas con las estrategias cognitivas que incluye las actividades $3,7,11,15 \mathrm{y}$ 19. La número 3 indica que los estudiantes siempre intentan descubrir las ideas princi- pales o la información relevante de la tarea o actividad de aprendizaje (3). En segundo lugar, muchas veces se preguntan cómo se relaciona la información importante de la actividad con lo que ya saben (7). 


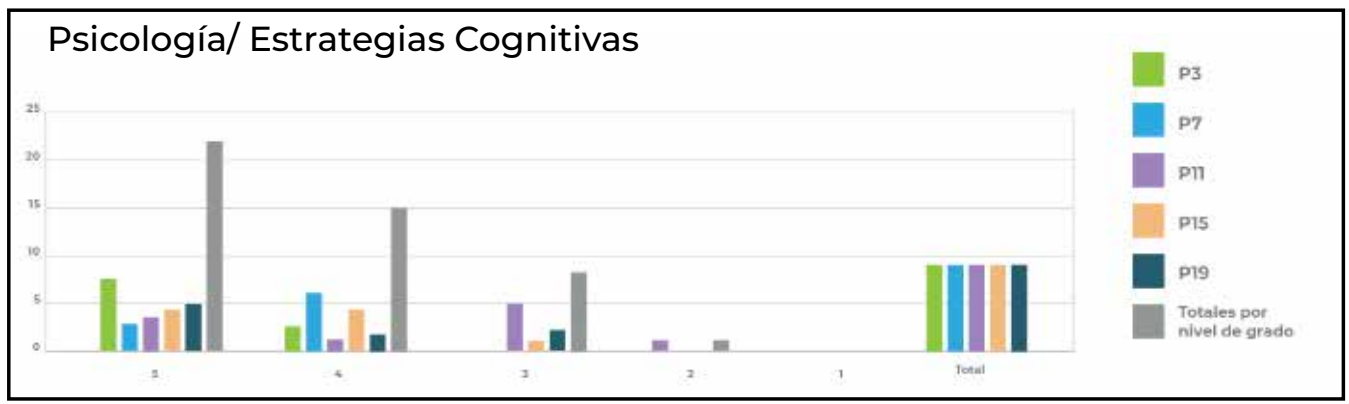

Gráfico 3. Distribución del grado de frecuencia de las estrategias cognitivas. Carrera de Psicología.

El Gráfico 4 agrupa las actividades relacionadas con las estrategias de planificación que incluyen las actividades 4,8 , 12,16 y 20. La mayoría de los estudiantes expresan que siempre intentan concretar qué se les pide en la tarea y se esfuerzan por comprender la información clave de la actividad antes de intentar resolverla (8 y 20). Igualmente, la misma cantidad, cinco, indican que muchas veces intentan descubrir las ideas principales o la información relevante de dicha tarea o actividad (4).

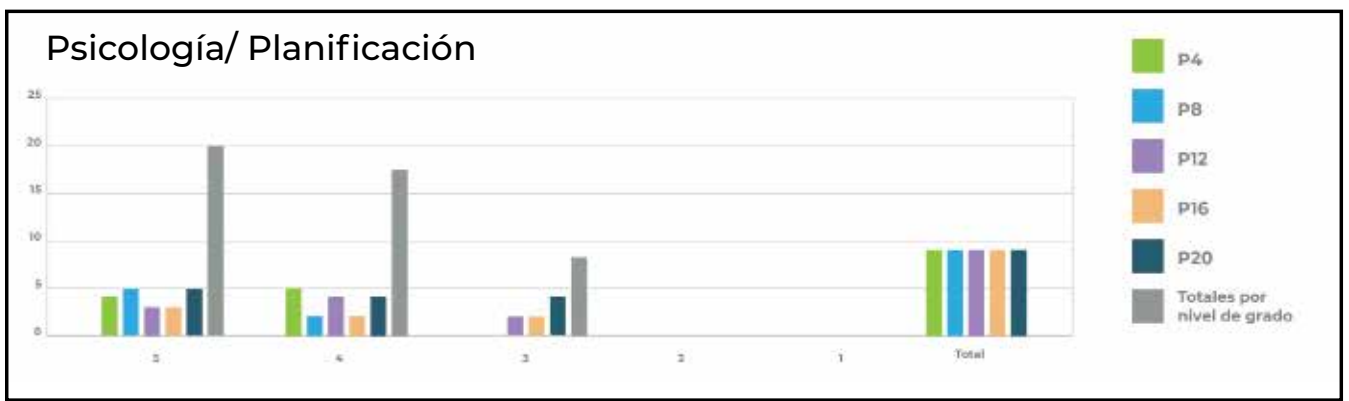

Gráfico 4. Distribución del grado de frecuencia de las estrategias de planificación. Carrera de Psicología.

En el Gráfico 5 se representa la distri- lo están haciendo (2). La misma cantidad bución del grado de frecuencia en que se de estudiantes reporta que muchas veces aplican las actividades de monitoreo $(2,6$, comprueban su precisión a medida que 10, 14 y 18). La mayoría, seis, señala que avanzan en la realización de la actividad siempre comprueban su trabajo mientras (18).

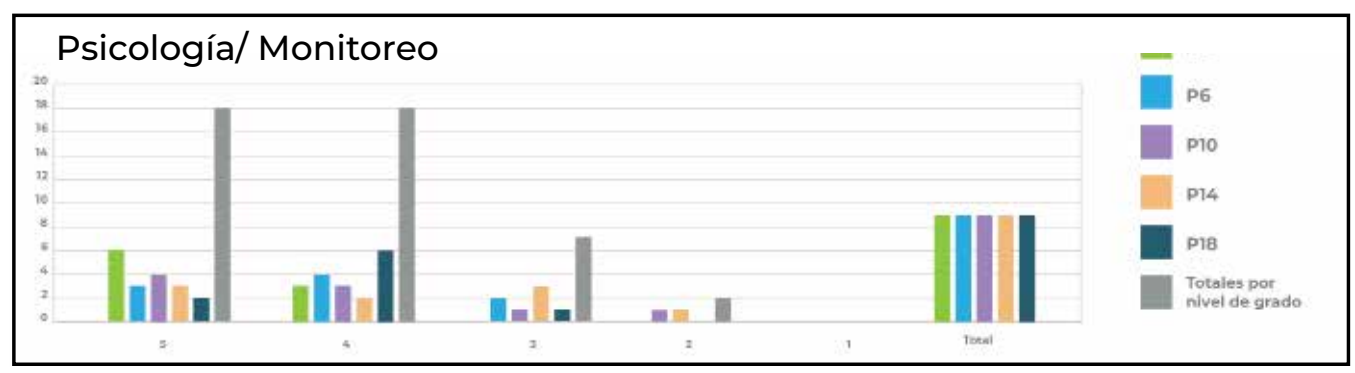

Gráfico 5. Distribución del grado de frecuencia de las estrategias de monitoreo. Carrera de Psicología. 
El Gráfico 6 presenta los resultados sobre el grado de frecuencia de aplicación de las estrategias metacognitivas por los estudiantes de la Carrera de Administración de Empresas. Se observa que mayoría se- lecciona las diez primeras actividades en igual proporción en el grado 5 (siempre). Predominan las actividades de conciencia metacognitiva y monitoreo con tres actividades cada una.

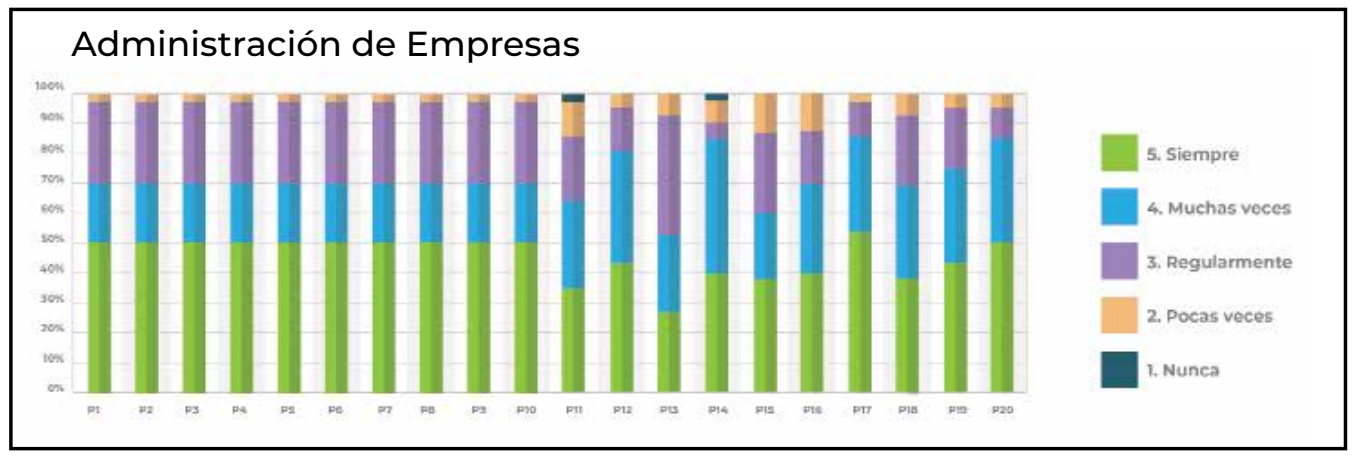

Gráfico 6. Distribución de respuestas por actividad y grado de frecuencia del inventario de estrategias metacognitivas. Carrera de Administración de Empresas

El Gráfico 7 agrupa las actividades relacionadas con la conciencia metacognitiva que incluye las actividades 1, 5, 9, 13 y 17 . La mayoría de los estudiantes, 34, siem- pre están conscientes de su esfuerzo por intentar comprender la actividad antes de empezar a resolverla (17).

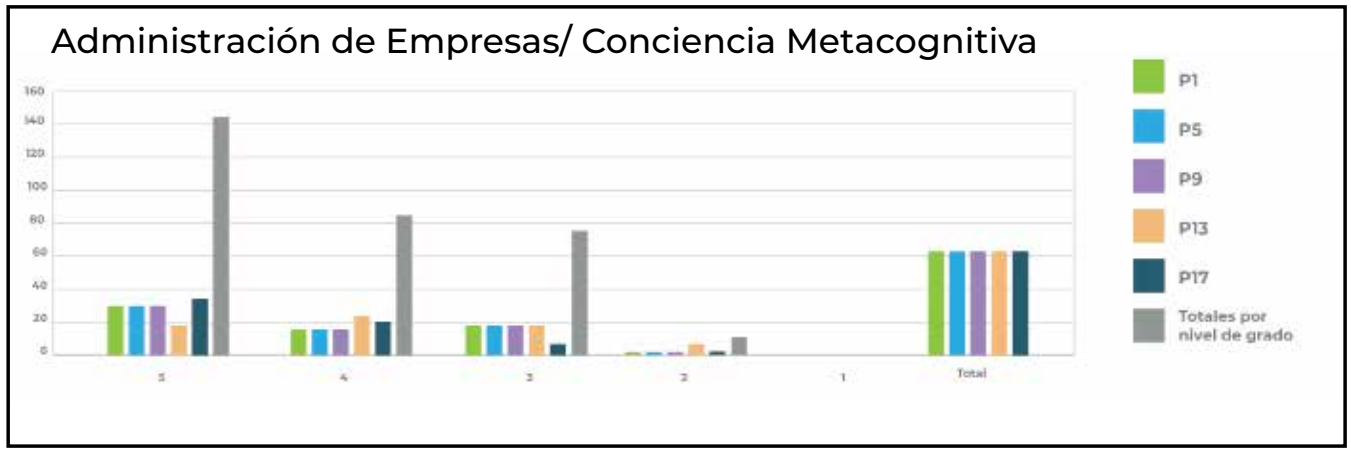

Gráfico 7. Distribución del grado de frecuencia de las estrategias de conciencia metacognitiva. Carrera de Administración de Empresas.

En el Gráfico 8 se representan los resultados del grado de frecuencia de las estrategias cognitivas que consisten en las actividades $3,7,11,15$ y 19 . La mayoría de los estudiantes, 31 , considera que siempre intentan descubrir las ideas principales o la información relevante de dicha tarea o actividad y se preguntan cómo se relaciona la información importante de la actividad con lo que ya saben ( 3 y 7 ). 


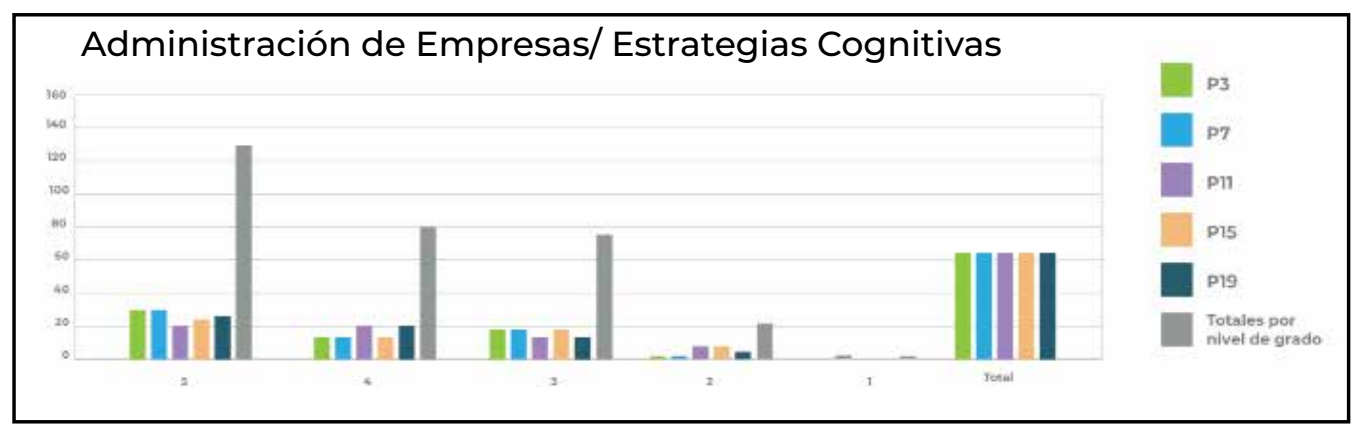

Gráfico 8. Distribución del grado de frecuencia de las estrategias cognitivas. Carrera de Administración de Empresas

El Gráfico 9 representa los resultados de los estudiantes, 32, expresan que siempre las actividades relacionadas con las estra- se esfuerzan por comprender la informategias de planificación que incluyen las ac- ción clave de la actividad antes de intentar tividades 4, 8, 12, 16 y 20. La mayoría de resolverla (20).

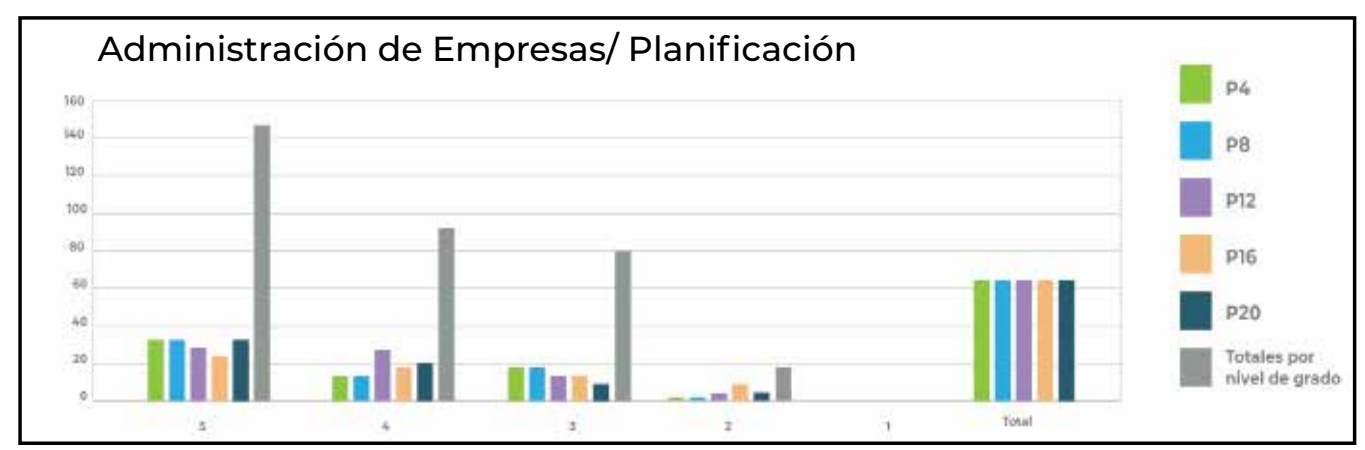

Gráfico 9. Distribución de frecuencia de las estrategias de planificación. Carrera de Administración de Empresas

En el Gráfico 10 se representa la distribución del grado de frecuencia en que aplican las actividades de monitoreo $(2,6,10$, 14 y 18). La mayoría, 31 , señala que siempre comprueban su trabajo mientras lo están haciendo, identifican y corrigen sus errores y una vez finalizada la actividad, son capaces de reconocer lo que dejaron sin realizar $(2,6$, y 10$)$.

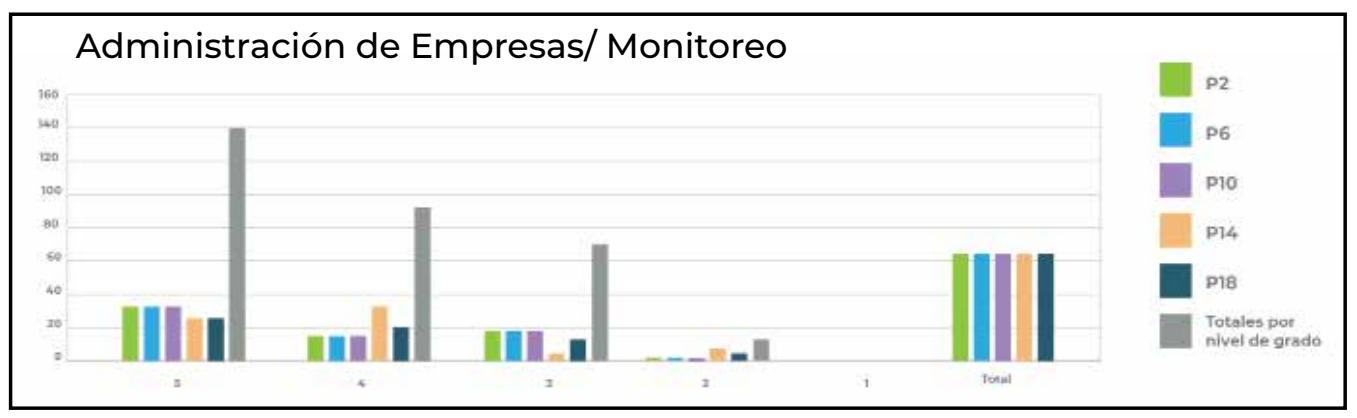

Gráfico 10. Distribución de frecuencias de las estrategias de monitoreo. Carrera de Administración de Empresas 


\section{DISCUSIÓN}

Los resultados muestran que la mayoría de los estudiantes de la Carrera de Psicología siempre intentan descubrir las ideas principales o la información relevante de la tarea o actividad que realizan, aplicando en este sentido una estrategia cognitiva. Por otra parte los estudiantes de la Carrera de Administración expresan que siempre son conscientes de lo que piensan sobre la actividad de aprendizaje o problema y que comprueban su trabajo mientras lo están realizando, demarcando una conciencia metacognitiva y la aplicación de estrategias de monitoreo. Las estrategias cognitivas pueden definirse como comportamientos planificados que seleccionan y organizan mecanismos cognitivos, afectivos y motrices con el fin de enfrentarse a situaciones-problema, globales o específicas, de aprendizaje (Muria, 1994, citado por Klimenko, 2009).

Klimenko y Alvares (2009) destacan "la importancia de la enseñanza de las estrategias cognitivas y metacognitivas con el fin de proporcionar a los estudiantes herra- mientas necesarias para convertirse en los aprendices autónomos y conscientes de su propio proceso de aprendizaje."

En cuanto a la conciencia metacognitiva Organista Díaz (2005) considera que la conciencia es parte del estado de conocimiento que va desde un nivel implícito a lo explicito, y la metacognición es parte del proceso, constituyéndose la conciencia como un componente más de la metacognición al igual que los factores estratégicos y de control.

Por tanto los resultados de este estudio son testimonio de lo que autores como Flavell (1979), Florez (2000), Livingston (2003) entre otros han sostenido que en el proceso de aprendizaje interactúan una serie de factores y actividades que fortalecen los procesos metacognitivos como las capacidad de desarrollar e implementar estrategias de transferencia del conocimiento, organización, autoevaluación, secuenciación de acciones, determinación y discriminación de la información.

\section{CONCLUSIONES}

Los resultados evidencian que la mayoría de respuestas (Total 24) abarcan de la Carrera de Psicología abarcan las actividades orientadas a la conciencia metacognitiva en el nivel 4 (muchas veces), mientras que en la Carrera de Administración de Empresas la mayoría (Total 145) se inclinan a actividades de planificación en el nivel 5 (siempre) como estrategias metacognitivas. En estas actividades el estudiante articula y define sus acciones de planificación de manera estratégica y determina las condiciones para activar el aprendizaje.

Tanto los estudiantes de Psicología como de Administración indican a través del inventario que están conscientes de qué técnica o estrategia de pensamiento usan y cuándo usarla y de los procesos de pensamiento que utilizan. Igualmente intentan descubrir las ideas principales o la información relevante de la tarea o actividad de aprendizaje, concretan lo qué se les pide en la tarea y se esfuerzan por comprender la información clave de la actividad antes de intentar resolverla, comprueban su trabajo mientras lo están haciendo.

Se debe acentuar que la metacognición constituye una fase fundamental del aprendizaje. El aprendizaje efectivo implica la planificación y el establecimiento de ob- 
jetivos, el seguimiento del progreso y la adaptación. Es importante tener presente que la capacidad de aprender de los estudiantes es adaptable, requiriendo del docente la planificación y desarrollo de ambientes, condiciones y oportunidades para propicias estrategias metacognitivas. La autorregulación comienza con el establecimiento de metas y la planificación, teniendo en cuenta las fortalezas y debilidades para activar la tarea de aprendizaje y la motivación para aprender. La verificación de las estrategias elegidas permite hacer ajustes y controla que los resultados estén alineados con los objetivos de aprendizaje.

Para subsecuentes estudios cabría realizar investigaciones para profundizar en especificidades respecto al tipo de estrategias de autorregulación acordes a las tareas o actividades y lograr verificar y describir las habilidades que desarrollan los estudiantes.

\section{BIBLIOGRAFÍA}

1. Arteta, H. y Huaire, E. (2016) Estrategias metacognitivas y concepciones de aprendizaje en estudiantes universitarios. Horizonte de la Ciencia 6 (11) $\mathrm{FE} /$ UNCP

2. MFlavell, J. H. (1979). Metacognition and cognitive monitoring: $\mathrm{A}$ new area of cognitive developmental inquiry. American Psychologist, 34, 906-911.

3. Flórez, R (2000) Autorregulación, Metacognición y Evaluación. Acción Pedagógica. Vol. 9, Nos. 1 y 2.

4. OKlimenko, Olena (2009) La enseñanza de las estrategias cognitivas y metacognitivas como una vía de apoyo para el aprendizaje autónomo en los niños con déficit de atención sostenida. Revista Virtual Universidad Católica del Norte [en linea] (Mayo-Agosto). Disponible en: http://www.redalyc.org/articulo. oa?id=194215432005
5. Klimenko, Olena, \& Alvares, José Luis. (2009). Aprender cómo aprendo: la enseñanza de estrategias metacognitivas. Educación y Educadores, 12(2), 11-28. Retrieved February 14,2019, from http://www. scielo.org.co/scielo.php?script $=$ sci_arttext\&pid=S0123-12942009000200003\&ln$\mathrm{g}=\mathrm{en} \&$ tlng $=\mathrm{es}$.

6. Pulido, A., Arcos, A., Pascual, R., y Garrido, V. (1988). Agresor, víctima e incidente de la violación: Un análisis descriptivo. Cuadernos de Política Criminal. 35, 291-316. 